\title{
Editorial \\ Special Issue: Advances in Architectural Acoustics
}

\author{
Nikolaos M. Papadakis ${ }^{1,2, *}$, Massimo Garai ${ }^{3}$ (D) and Georgios E. Stavroulakis ${ }^{1}$ (D) \\ 1 Institute of Computational Mechanics and Optimization (Co.Mec.O), School of Production Engineering and \\ Management, Technical University of Crete, 73100 Chania, Greece; gestavr@dpem.tuc.gr \\ 2 Department of Music Technology and Acoustics, Hellenic Mediterranean University, 74100 Rethymno, Greece \\ 3 Department of Industrial Engineering, University of Bologna, 40136 Bologna, Italy; massimo.garai@unibo.it \\ * Correspondence: nikpapadakis@isc.tuc.gr
}

check for

updates

Citation: Papadakis, N.M.; Garai, M.; Stavroulakis, G.E. Special Issue:

Advances in Architectural Acoustics. Appl. Sci. 2022, 12, 1728. https:// doi.org/10.3390/app12031728

Received: 8 December 2021

Accepted: 20 December 2021

Published: 8 February 2022

Publisher's Note: MDPI stays neutral with regard to jurisdictional claims in published maps and institutional affiliations.

Copyright: (C) 2022 by the authors. Licensee MDPI, Basel, Switzerland. This article is an open access article distributed under the terms and conditions of the Creative Commons Attribution (CC BY) license (https:// creativecommons.org/licenses/by/ $4.0 /)$.

\section{Introduction}

Satisfactory acoustics is crucial for the ability of spaces such as auditoriums and lecture rooms to perform their primary function. The acoustics of dwellings and offices greatly affects the quality of our life since we are all consciously or subconsciously aware of the sounds to which we are daily subjected. The aim of this special issue was to gather advances in architectural acoustics that hopefully could inspire researchers and acousticians to explore new directions in this age of scientific convergence and multidisciplinary cooperation.

The special issue was an exciting journey for us in which we had the opportunity to communicate with many people in the field from all over the world. Authors from Australia, Belgium, China, Finland, Greece, Italy, Japan, New Zealand, Spain, Sweden, Taiwan and the UK-including 19 universities and 4 acoustic firms and corporations-participated in this special issue.

Among the numerous submissions, 17 successfully passed the review process. For better presentation in this introductory text, the papers have been categorized as follows:

- Review studies;

- Historical Acoustics;

- Computational Acoustics;

- Design of concert or conference halls and open-plan offices;

- Miscellaneous (sound absorbers, listeners perception, machine learning).

\section{Review Studies}

\section{Italian-Style Opera Houses: A Historical Review}

In his work [1], D'Orazio investigated the historical development of Italian-style opera houses from the 16th century to the present day. Called "Italian" due to their origin, operas developed thanks to the mutual influence of the genre and the building characteristics. The acoustics of historical opera houses is now considered as intangible cultural heritage. The paper addresses the state-of-the-art literature-most of which is available in Italian - which can be driven easily by the sharing of historical and contemporary knowledge.

\section{Review of Acoustic Sources: Alternatives to a Dodecahedron Speaker}

In this study by Papadakis and Stavroulakis [2], fifteen acoustic sources alternative to a dodecahedron speaker are presented. Emphasis is placed on features such as omnidirectionality, repeatability, adequate sound pressure levels, even frequency response, accuracy in the measurement of acoustic parameters and the fulfillment of ISO 3382-1 requirements for sound sources. The collected data from this review can be used in many areas for the appropriate selection of an acoustic source according to the expected use. 


\section{Historical Acoustics}

3. Measurements of Acoustical Parameters in the Ancient Open-Air Theatre of Tyndaris (Sicily, Italy)

The outcomes of a measurement campaign of acoustical parameters in the ancient theatre of Tyndaris (Sicily) are presented in this paper by Astolfi et al. [3]. The results show that the reverberation time and sound strength values were relatively low when compared with other theatres because of the lack of the original architectural element of the scaenae frons. When combining this effect with the obvious condition of an unroofed space, issues emerge in terms of applicability of the protocols recommended in the ISO standard.

\section{Evolutionary Analysis of the Acoustics of the Baroque Church of San Luis De Los Franceses (Seville)}

The church of San Luis de los Franceses, built by the Jesuits for their novitiate in Seville (Spain), is an example of a Baroque church with a central floor plan. The acoustics of this church were studied by Alberdi et al. [4] through in situ measurements and virtual models. The main objective was to analyze the evolution and perception of its sound field from the 18th to 21st centuries, considering the different audience distributions and sound sources as well as the modifications in furniture and coatings.

\section{Historically Based Room Acoustic Analysis and Auralization of a Church in the 1470s}

This paper by Autio et al. [5] describes the historical acoustics of an important abbey church in Sweden in the 1470s. A digital historical reconstruction was developed, liturgical material specific to this location was recorded and auralized and a room acoustic analysis was performed. The analysis was guided by the liturgical practices in the church and the monastic order connected to it.

\section{Computational Acoustics}

6. Potential of Room Acoustic Solver with the Plane-Wave Enriched Finite Element Method

A preliminary study on the partition of unity finite element method (PUFEM) as a room acoustic solver was presented by Okuzono et al. [6]. The PUFEM performance was examined against a standard FEM in a single room and a coupled room, including frequency-dependent complex impedance boundaries of Helmholtz resonator-type sound absorbers and porous sound absorbers. The results demonstrated that the PUFEM could accurately predict wideband frequency responses under a single coarse mesh with considerably fewer degrees of freedom than the standard FEM.

\section{Time Domain Room Acoustic Solver with a Fourth-Order Explicit FEM Using Modified Time Integration}

This paper by Yoshida et al. [7] presents a proposal of a time domain room acoustic solver using a novel fourth-order accurate explicit time domain finite element method (TD-FEM). The proposed method could use irregularly shaped elements whilst maintaining fourth-order accuracy in time without an additional computational complexity compared with the conventional method. The practicality of the method at kilohertz frequencies was presented via two numerical examples of acoustic simulations in a rectangular sound field, including complex sound diffusers and in a concert hall with a complex shape.

8. An Energy Model for the Calculation of Room Acoustic Parameters in Rectangular Rooms with Absorbent Ceilings

In this paper by Nilsson and Arvidsson [8], a statistical energy analysis (SEA) model was derived where a non-isotropic sound field was considered. The sound field was subdivided into a grazing and a non-grazing part, where the grazing part referred to waves propagating almost parallel to the suspended ceiling. A comparison with measurements was performed for a classroom configuration, which revealed that the new model agreed better with the measurements than the classical one (diffuse field). 
9. Coherent Image Source Modeling of Sound Fields in Long Spaces with a Sound-Absorbing Ceiling

This paper by Min and Xu [9] presents a coherent image source model for a simple yet accurate prediction of the sound field in long enclosures with a sound-absorbing ceiling. In the proposed model, the reflections on the absorbent boundary were separated from those on the reflective ones when evaluating the reflection coefficients. The model was compared with the classic wave theory, an existing coherent image source model and a scale model experiment.

10. Power Response and Modal Decay Estimation of the Room Reflections from Spherical Microphone Array Measurements Using Eigenbeam Spatial Correlation Model

The application of the eigenbeam spatial correlation method in estimating the time frequency-dependent directional reflection powers and model decay times was presented by Bastine et al. [10]. The experimental results evaluated the application of the proposed technique for two rooms with distinct environments using their room impulse response measurements recorded by a spherical microphone array. The experimental observations proved that the proposed model is a promising tool in characterizing early and late reflections, which is beneficial in controlling the perceptual factors of room acoustics.

\section{Design of Concert or Conference Halls and Open-Plan Offices}

11. On the Sequence of Unmasked Reflections in Shoebox Concert Halls

This work by García Gómez et al. [11] is a tribute to the 90th anniversary of Sir Harold Marshall and his early innovative ideas of the acoustic signature of a hall. By analyzing the cross-sections of three concert halls, this study quantified the potential links between the architectural form of a hall, the resultant skeletal reflections and the properties of its acoustic signature. Whilst doing so, this study identified potential masking reflections through a visual and an analytical assessment of the skeletal reflections.

\section{Comfort Distance-A Single-Number Quantity Describing Spatial Attenuation in Open-Plan Offices}

This study by Hongisto and Keränen [12] introduces a new single-number quantity, the comfort distance $r_{C}$, that integrates the quantities of the A-weighted sound pressure

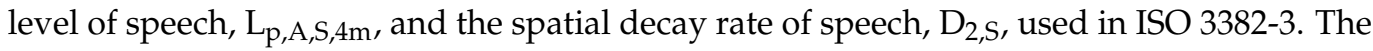
new quantity describes the distance from an omnidirectional loudspeaker where the Aweighted sound pressure level of normal speech falls below $45 \mathrm{~dB}$. The study explains why the comfort criterion level is set to $45 \mathrm{~dB}$ and explores the comfort distances in 185 offices reported in previous studies.

\section{A Trial Acoustic Improvement in a Lecture Hall with MPP Sound Absorbers and FDTD Acoustic Simulations}

The feasibility and performance of micro-perforated panels (MPPs) when used as an acoustic treatment in lecture rooms were investigated in this work by Cingolani et al. [13]. Three different micro-perforated steel specimens were first designed following existing predictive models and then physically manufactured through 3D additive metal printing. Numerical simulations were carried out using a full-spectrum wave-based method: a finite-difference time-domain (FDTD) code was chosen to better handle time-dependent signals as the verbal communication. The outcomes of the process showed the influence of the acoustic treatment in terms of the reverberation time $\left(\mathrm{T}_{30}\right)$ and sound clarity $\left(\mathrm{C}_{50}\right)$.

\section{Mechanism Analysis of the Influence of Seat Attributes on the Seat Dip Effect in Music Halls}

In this paper [14], Min and Liao performed numerical simulations on the basis of the finite element method to study the influence of seat attributes (seat height, seat spacing and seat absorption) on the seat dip effect (SDE) and the corresponding mechanism. The mapping of the sound spatial distribution related to the SDE was employed to observe the behavior of sound between the seats. A mechanism analysis revealed that the SDE 
was highly associated with standing waves inside the seat gaps and with the "diffusion" effect on the grazing incident waves by the energy flow vortexes around the top surfaces of the seats.

\section{Miscellaneous (Sound Absorbers, Listeners Perception, Machine Learning) \\ 15. A Parametric Study of the Acoustic Performance of Resonant Absorbers Made of Micro-perforated Membranes and Perforated Panels}

The paper by Pan and Martellotta [15] first investigated the reliability of prediction models for perforated and micro-perforated panels by a comparison with measured data. Subsequently, whilst taking advantage of a parametric optimization algorithm, it was shown how to design an absorber covering three octave bands from $500 \mathrm{~Hz}$ to $2 \mathrm{kHz}$ with an average sound absorption coefficient of approximately 0.8 . Such a solution might be conveniently realized whilst using optically transparent panels, which might offer extra value as they could ensure visual contact whilst remaining neutral in terms of the design.

16. Listeners Sensitivity to Different Locations of Diffusive Surfaces in Performance Spaces: The Case of a Shoebox Concert Hall

The effects of diffusive surfaces on the acoustic design parameters in a real shoebox concert hall with variable acoustics (Espace de Projection, IRCAM, Paris, France) were investigated by Shtrepi et al. [16]. Acoustic measurements were performed in six hall configurations by varying the location of the diffusive surfaces over the front, mid and rear part of the lateral walls whilst the other surfaces were maintained absorptive or reflective. Conventional ISO 3382 objective acoustic parameters were evaluated along with a subjective investigation performed by using the ABX method with auralization at two listening positions.

\section{A Machine Learning Based Prediction Model for the Sound Absorption Coefficient of a Micro-Expanded Metal Mesh (MEMM)}

The objective of this study by Tsay and Yeh [17] was to develop a prediction model for a MEMM via a machine learning approach. An experiment including 14 types of MEMM was first performed in a reverberation room measured according to ISO 354. To predict the sound absorption coefficient of the MEMM, the capability of three conventional models and three machine learning (ML) models of the supervised learning method were studied for the development of the prediction model.

\section{Conclusions}

The diversity and richness of the presented papers demonstrates the liveliness of the research in the field of architectural acoustics. In this exciting context, it is easy to guess that more novel ideas are yet to appear along with further research and new applications. We hope that this collection will serve as an inspiration for our fellow acousticians, especially the young ones, to explore new ways in architectural acoustics in the future.

Acknowledgments: We are grateful to all contributors who made this special issue a success. First of all, to the authors who trusted their work to us. Thank you all, one by one. We express our gratitude to the Applied Sciences editorial team for their effective communication and tireless work. We would like to personally thank our assistant editor, Enoch Li, for unlimited help, kindness and understanding. Enoch, thank you so much for your help and positive spirit, even after numerous exchanges of emails to resolve small or big issues. We also thank our initial assistant editor, Luca Shao. Finally, we extend our thanks to all reviewers, who made it possible to evaluate and select the excellent works presented in this special issue.

Conflicts of Interest: The authors declare no conflict of interest. 


\section{References}

1. D'Orazio, D. Italian-Style Opera Houses: A Historical Review. Appl. Sci. 2020, 10, 4613. [CrossRef]

2. Papadakis, N.M.; Stavroulakis, G.E. Review of Acoustic Sources Alternatives to a Dodecahedron Speaker. Appl. Sci. $2019,9,3705$. [CrossRef]

3. Astolfi, A.; Bo, E.; Aletta, F.; Shtrepi, L. Measurements of Acoustical Parameters in the Ancient Open-Air Theatre of Tyndaris (Sicily, Italy). Appl. Sci. 2020, 10, 5680. [CrossRef]

4. Alberdi, E.; Galindo, M.; León-Rodríguez, Á.L. Evolutionary Analysis of the Acoustics of the Baroque Church of San Luis de los Franceses (Seville). Appl. Sci. 2021, 11, 1402. [CrossRef]

5. Autio, H.; Barbagallo, M.; Ask, C.; Bard Hagberg, D.; Lindqvist Sandgren, E.; Strinnholm Lagergren, K. Historically Based Room Acoustic Analysis and Auralization of a Church in the 1470s. Appl. Sci. 2021, 11, 1586. [CrossRef]

6. Okuzono, T.; Mohamed, M.S.; Sakagami, K. Potential of room acoustic solver with plane-wave enriched finite element method. Appl. Sci. 2020, 10, 1969. [CrossRef]

7. Yoshida, T.; Okuzono, T.; Sakagami, K. Time domain room acoustic solver with fourth-order explicit FEM using modified time integration. Appl. Sci. 2020, 10, 3750. [CrossRef]

8. Nilsson, E.; Arvidsson, E. An energy model for the calculation of room acoustic parameters in rectangular rooms with absorbent ceilings. Appl. Sci. 2021, 11, 6607. [CrossRef]

9. Min, H.; Xu, K. Coherent Image Source Modeling of Sound Fields in Long Spaces with a Sound-Absorbing Ceiling. Appl. Sci. 2021, 11, 6743. [CrossRef]

10. Bastine, A.; Abhayapala, T.D.; Zhang, J.A. Power Response and Modal Decay Estimation of Room Reflections from Spherical Microphone Array Measurements Using Eigenbeam Spatial Correlation Model. Appl. Sci. 2021, 11, 7688. [CrossRef]

11. Gómez, J.Ó.G.; Wright, O.; van den Braak, B.; Sanz, J.; Kemp, L.; Hulland, T. On the Sequence of Unmasked Reflections in Shoebox Concert Halls. Appl. Sci. 2021, 11, 7798. [CrossRef]

12. Hongisto, V.; Keränen, J. Comfort Distance-A Single-Number Quantity Describing Spatial Attenuation in Open-Plan Offices. Appl. Sci. 2021, 11, 4596. [CrossRef]

13. Cingolani, M.; Fratoni, G.; Barbaresi, L.; D'orazio, D.; Hamilton, B.; Garai, M. A Trial Acoustic Improvement in a Lecture Hall with MPP Sound Absorbers and FDTD Acoustic Simulations. Appl. Sci. 2021, 11, 2445. [CrossRef]

14. Min, H.; Liao, Y. Mechanism Analysis of the Influence of Seat Attributes on the Seat Dip Effect in Music Halls. Appl. Sci. 2021, 11, 9768. [CrossRef]

15. Pan, L.; Martellotta, F. A parametric study of the acoustic performance of resonant absorbers made of micro-perforated membranes and perforated panels. Appl. Sci. 2020, 10, 1581. [CrossRef]

16. Shtrepi, L.; Di Blasio, S.; Astolfi, A. Listeners sensitivity to different locations of diffusive surfaces in performance spaces: The case of a shoebox concert hall. Appl. Sci. 2020, 10, 4370. [CrossRef]

17. Tsay, Y.-S.; Yeh, C.-Y. A Machine Learning Based Prediction Model for the Sound Absorption Coefficient of Micro-Expanded Metal Mesh (MEMM). Appl. Sci. 2020, 10, 7612. [CrossRef] 